\title{
Pressure and Temperature Dependence of the Longitudinal Proton and Deuteron Relaxation Rates in $\mathrm{NH}_{3}$ and $\mathrm{ND}_{3}$
}

\author{
H. Hauer, E. Lang, and H.-D. Lüdemann
}

Institut für Biophysik und Physikalische Biochemie, Universität Regensburg, Postfach 397, D-8400 Regensburg

\section{Flüssigkeiten / Hohe Drücke / Magnetische Kernresonanz / Transporterscheinungen}

The molecular mobility of liquid ammonia is derived from the determination of the longitudinal relaxation times of the protons and deuterons. The experiments were performed in the temperature interval between the melting pressure curve and $467 \mathrm{~K}_{\text {for }} \mathrm{NH}_{3}$ and to $351 \mathrm{~K}$ for $\mathrm{ND}_{3}$ at pressures up to $250 \mathrm{MPa}$. At temperatures below $\sim 350 \mathrm{~K}$ the molecular mobility can be described by the isotropic smallstep diffusion model. The activation energies at constant pressure are derived for the rotatoric diffusion to $7.0 \pm 0.5 \mathrm{~kJ} \cdot \mathrm{mol}^{-1}$ and for the translatoric diffusion to $6 \pm 1 \mathrm{~kJ} \cdot \mathrm{mol}^{-1}$ from the temperature dependence of the relaxation rates. In addition the activation encrgy at constant volume for the rotatoric diffusion has been determined to $5.7 \pm 0.5 \mathrm{~kJ} \cdot \mathrm{mol}^{-1}$. The isotherms for all relaxation rates are linear in a $\log \left(1 / T_{1}\right)$ versus $1 / T$ plot, yielding $\Delta V_{\text {intra }}^{*}=2.5 \pm 0.6 \mathrm{~cm}^{3} \cdot \mathrm{mol}^{-1}$ and $\Delta V_{\text {inte }}^{*}=5 \pm 1 \mathrm{~cm}^{3} \cdot \mathrm{mol}^{-1}$.

Die molekulare Beweglichkeit des flüssigen Ammoniaks wurde mit Hilfe longitudinaler Relaxationszeitmessungen der Protonen und Deuteronen untersucht. Die Messungen wurden im Temperaturbereich zwischen der Schmelzdruckkurve und $467 \mathrm{~K}_{\text {für }} \mathrm{NH}_{3}$ bzw. $351 \mathrm{~K}$ für $\mathrm{ND}_{3}$ bei Drücken bis $250 \mathrm{MPa}$ vorgenommen. Bei Temperaturen $\leqslant 350 \mathrm{~K}$ läßt sich die molekulare Beweglichkeit durch ein isotropes small-step Diffusionsmodell beschreiben. Aus der Temperaturabhängigkeit der Relaxationsraten wurden die Aktivierungsenergien bei konstantem Druck für die rotatorische Diffusion zu $7,0 \pm 0,5 \mathrm{~kJ} \cdot \mathrm{mol}^{-1}$ und für die translatorische Diffusion zu $6 \pm 1 \mathrm{~kJ} \cdot \mathrm{mol}^{-1}$ bestimmt. Für die rotatorische Diffusion wurde die Aktivierungsenergie bei konstantem Volumen zu $5,7 \pm 0,5 \mathrm{~kJ} \cdot \mathrm{mol}^{-1}$ errechnet. Die Isothermen für alle Relaxationsraten verlaufen in einer $\log 1 / T_{1}$ gegen $1 / T$ Darstellung linear. $\left(\Delta V_{\text {inira }}^{*}=2.5 \pm 0.6 \mathrm{~cm}^{3} \cdot \mathrm{mol}^{-1}\right.$. $\left.\Delta V_{\text {inter }}^{*}=5 \pm 1 \mathrm{~cm}^{3} \cdot \mathrm{mol}^{-1}\right)$

\section{Introduction}

The hydrides of nitrogen, oxygen, and fluorine form liquids with rather unique structures. The physical properties of these liquids are normally explained by the ability of these three hydrides to participate in hydrogen bonding with their hydrogen atoms as well as with the lone electron pairs. The pronounced hydrogen bonding in liquid water is well established [1]. As derived from small angle X-ray and neutron scattering liquid ammonia however does possess a significantly different radial distribution function with approximately 12 next neighbours [2] as compared to the 4-5 next neighbours of water.
Though liquid ammonia has been the subject of several investigations, it is still the subject of discussion whether the dynamic properties of this liquid reveal significant hydrogen bonding, and different authors have derived from their data pronounced hydrogen bonding $[3,4]$ as well as no contribution at all from this interaction $[5,6]$.

As well known, NMR presents a powerful tool for the study of molecular motions in liquids. Valuable information can be gained from the temperature- and pressure dependence of the spin-lattice relaxation times. Previous work has investigated the temperature dependence of the relaxation times of $\mathrm{H}[7]$, ${ }^{14} \mathrm{~N}[3,8]$ and ${ }^{15} \mathrm{~N}[4]$ in $\mathrm{NH}_{3}$ and $\mathrm{D}[8,9],{ }^{14} \mathrm{~N}[8]$, and ${ }^{15} \mathrm{~N}[4]$ in liquid $\mathrm{ND}_{3}$. In this paper the pressure dependence 
of the relaxation rates of $\mathrm{H}$ in $\mathrm{NH}_{3}$ and $\mathrm{D}$ in $\mathrm{ND}_{3}$ is presented. In the PT-region studied, the relaxation of the deuterons in $\mathrm{ND}_{3}$ is completely determined by the quadrupole relaxation mechanism. In the low temperature range the proton relaxation is dominated by the dipole-dipolle mechanism, at higher temperatures the spin-rotation me:chanism contributes significantly to the observed relaxation rate.

\section{Experimenital}

The deuteron and proton longitudiinal relaxation times $T_{1}$ were obtained on a Varian XL 100-15 Ft NNMR spectrometer interfaced to a $16 \mathrm{~K}$ Varian $620 \mathrm{~L}-100$ computer with disk accessory by a $t_{1}-90^{\circ}-t_{2}-180^{\circ}-t_{1}-90^{\circ}$ pulse sequence. The observe frequency on this instrument is $100.1 \mathrm{MHz}$ for proitons and $15.4 \mathrm{MHz}$ for deuterons. A modified variable temperature accessory of this spectrometer was used in the experiments. The temperatures were determined to $\pm 0.5 \mathrm{~K}$ with a metal sheathed miniature chromel-alumel thermocouple. Deuteroammonia ( $99 \%$ deuterated) was purchased from Sharp and Dohme, München.

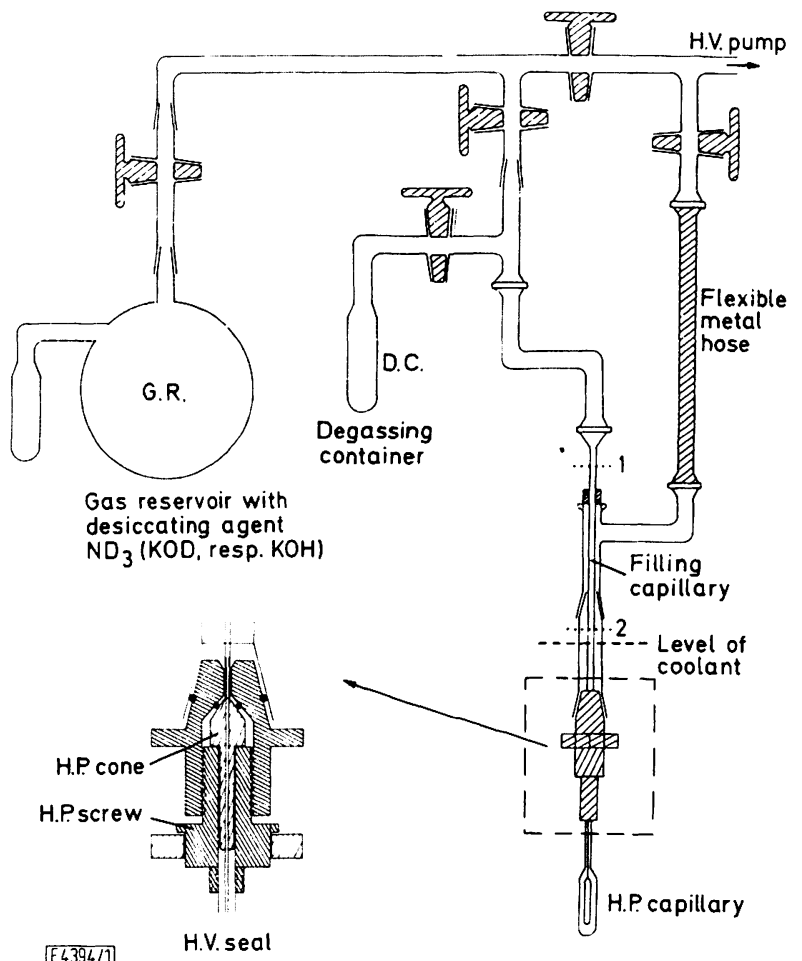

Fig. 1

High vacuum apparatus for the filling of oxygen free low boiling substances into the high pressure capillaries. (For functional details see text)

The high pressure equipment used has been described previously [10]. Oxygen-free dry ammonia was prepared in the high vacuum apparatus described in Fig. 1. A proper quantity of dried ammonia was condensed from the reservoir into the degassing container (D.C.) by cooling this container with acetone/carbondioxyde mixtures. The sample was thoroughly degassed by at least five freeze-pumpe-thaw cycles to a final pressure of $7 \cdot 10^{-3} \mathrm{~Pa}$. Freezing was accomplished by immersing the container (D.C.) into liquid nitrogen, the melting of the ammonia was done in acetone/carbondioxyde mixtures. After degassing of the ammonia the high pressure capillary was immersed in a methylcyclohexane bath to the level indicated in Fig. 1 and cosled to $205 \mathrm{~K}$. All connections to the high vacuum line were shut off and the ammonia in the degassing container allowd to warm up. After sufficient ammonia had condensed into te high pressure capillary, the filling glass capillary was flame sealed tt position 1. All glass parts and the high vacuum seal (H.V.S.) wee removed under continuous cooling from the high pressure caillary and the filling capillary was resealed at position 2 . The fecooled copper beryllium cell was screwed onto the high pressur cell, while the assembly remained in the cold methylcyclohexare. The whole assembly was connected to the pressure. generatng equipment and a pressure of $20 \mathrm{MPa}$ was applied. Afterwads the whole assembly was removed from the cold methylcycloexane bath.

This elaborateprocedure was necessary for two reasons:

1. The Teflon shrnk hose, which separates the liquid under study from the pressire generating liquid is not sufficiently vacuum tight to permitthe filling of oxygen-free liquid.

2. The low boilin, point of ammonia demands that all filling and sealing operatins are done at temperatures well below $230 \mathrm{~K}$.

As controlled by repeated $T_{1}$-measurements under identical conditions, the anmonia in properly filled and assembled cells remains free of aly contamination for several months.

\section{Theory}

The main relixation mechanisms for proton and deuteron relaxation are thedirect dipole-dipole interaction, the spin-rotation interaction and the quadrupole interaction.

\section{Dipole-Dipole and Spin-Rotation Relaxation for Protons}

The dipole-diple interaction contains two contributions due to the interaction of the nuclei within the same molecule and due to nuclei on differnt molecules. The intramolecular relaxation rate $\left(1 / T_{1}\right)_{\text {intra }}$ is due $\mathrm{o}$ changes in the orientation of the vector $\boldsymbol{r}_{12}$ connecting two ruclei within the same molecule, whereas the intermolecular relixation rate $\left(1 / T_{1}\right)_{\text {inter }}$ is due to changes in length and orientation if $\boldsymbol{r}_{12}$ between nuclei on different molecules. For a liquid conssting of isotropically reorienting molecules the intramolecular reaxation rate is under extreme narrowing conditions given by $[1-14]$

$\left(\frac{1}{T_{1}}\right)_{\text {intra }}=\frac{3}{2} \sum_{i>j} \frac{\gamma^{4} \hbar^{2}}{r_{i j}^{6}} \tau_{\theta, \text { eff }}^{(2)}$

where the sum rurs over all spin pairs that contribute to the relaxation of a single spir and $\tau_{\theta, \text { eff }}^{(2)}$ is the zero frequency Fourier transform of the correspondng auto-correlation function. For a symmetric top $\tau_{\theta \text {,eff }}^{(2)}$ is given in the Refs. $[13,15]$.

The intermolecular dipolar contribution is generally calculated assuming modulation of the dipole-dipole interaction by translational diffusion cnly. This gives [11]

$$
\left(\frac{1}{T_{1}}\right)_{\text {inter }}=\frac{6 \pi^{2}}{5} r^{2} \gamma^{4} \frac{N \cdot \eta}{T} \text {. }
$$

Various modifications of this equation have been given $[16,20]$. In the following cnly the proportionality between $\left(1 / T_{1}\right)_{\text {inter }}$ and $(\rho \cdot \eta) / T$ will be used.

The spin-rotation interaction is caused through a magnetic field produced by rotatons of the charge distribution in the vicinity of a given nucleus [21]. The relaxation rate caused by this mechanism is usually given by $[12,21]$.

$$
\left(\frac{1}{T_{1}}\right)_{\mathrm{SR}}=\frac{2 k T}{3 \hbar^{2}} \tau_{J} \sum_{i, j} I_{j} C_{i j}^{2} \text {. }
$$

In this equation it is assumed that no correlation exists between the orientation and the angular momentum. In the axis system of the moment of inertia tensor the latter is equivalent to the angular velocity correlation function, which is the actual quantity of interest in liquids. Further it is assumed that $\tau_{J j}=\tau_{J}$. The spin-rotation coupling tensor $c$ for the protons in $\mathrm{NH}_{3}$ contains non-diagonal 
elements $\mathrm{n}$ the principal inertial axis system [:]. Its elements can be ottained from microwave data $[23,24]$.

\section{Quadrupole Interaction}

The quadrupole relaxation rate is in the extrem narrowing limit and with an axially symmetric field gradient tensr given through the relation

$\left(\frac{1}{T_{1}}\right)_{Q}=\frac{3}{8}\left(\frac{e^{2} q Q}{\hbar}\right)^{2} \tau_{\theta, \text { eff }}^{(2)}$.

The correlation time $\tau_{\theta \text { eff }}^{(2)}$ is given by $[15,16]$ vith $\theta$ the angle between :he symmetry axis of the molecule and ie symmetry axis of the field gradient tensor.

\section{Separation of the Different Contributions of ae Proton Relaxation Times $T_{1}$}

Provided the quadrupole coupling constant QCC) is known for a given molecule in the liquid, the reorientcional correlation time $\tau_{\theta, \text { erf }}^{(2)}$ is directly determined from the expernental relaxation rate. Atkins et al. [8] showed for the case of liqid ammonia that the correlation times $\tau_{\theta \text { eff }}^{(2)}$ transform under isotoic substitution as

$\frac{\tau_{\theta}\left(\mathrm{NH}_{3}\right)}{\tau_{\theta}\left(\mathrm{ND}_{3}\right)}=\left(\frac{I\left(\mathrm{NH}_{3}\right)}{I\left(\mathrm{ND}_{3}\right)}\right)^{1 / 2}$

$I=$ moment of inertia

Thus one can get directly the correlation tme for the intramolecular dipolar relaxation rate in $\mathrm{NH}_{3}$ frim the measured deuteron relaxation times of $\mathrm{ND}_{3}$. In the rotatimal diffusion limit the reorientational correlation time $\tau_{\theta \text { eff }}^{(2)}$ and the argular momentum correlation time $\tau_{J}$ are related by $[15,25]$

$\tau_{\theta, \text { eff }}^{(2)} \cdot \tau_{J}=\frac{I_{\perp}}{6 k T} \cdot f_{2}(\theta, \alpha), \quad \alpha=\frac{I_{\lrcorner}}{I_{H}}$,

$f_{2}(\theta, \alpha)=\frac{\left(3 \cos ^{2} \theta-1\right)^{2}}{4}+\frac{18 \sin ^{2} \theta \cos ^{2} \theta}{5+\alpha}+\frac{9}{4} \frac{\sin ^{4} \theta}{1+2 \alpha}$

$I_{\lrcorner}, I_{\|}$- component of the moment of inertia tersor.

With $\tau_{J}$ thus obtained, $\left(1 / T_{1}\right)_{\text {SR }}$ can be calcuated with Eq. (5). Finally the intermolecular relaxation rate is given by

$\left(\frac{1}{T_{1}}\right)_{\text {inter }}=\left(\frac{1}{T_{1}}\right)_{\text {exp }}-\left(\frac{1}{T_{1}}\right)_{\text {intra }}-\left(\frac{1}{T_{1}}\right)_{\mathrm{SR}}$.

\section{Results and Discussion}

\section{Estimate of the QCC of Deuteroammonia in the Liquid State}

From the determination of the longitudinal proton and deuteron relaxation times in liquid $\mathrm{NH}_{3}$ and $\mathrm{ND}_{3}$ Powles et al. $[6,7,9]$ derived the QCC as $\left(e^{2} q Q\right) / h=245 \pm 25 \mathrm{kHz}$ assuming $\tau_{\theta}^{\mathrm{DD}}=\tau_{\theta}^{\mathrm{Q}}$. Applying instead Eq. (5) proposed by Atkins et al. [8] yields: $\left(e^{2} q Q\right) / h=208 \pm 21 \mathrm{kHz}$. As can be seen from a comparison of the corresponding nitrogen- 14 $T_{1}$ data of ${ }^{14} \mathrm{NH}_{3}$ and ${ }^{14} \mathrm{ND}_{3}[3,8]$ for this nucleus the decrease of the QCC in going from the gas phase to the solid state is much smaller, and thus QCC of the liquid can be estimated with considerably higher accuracy (see Table I), the latter value is obviously the more realistic choice (see also [26]).

\section{Relaxation Times at Saturation Pressure}

The spin-lattice relaxation times at saturation pressure have been measured over the temperature range of $197 \mathrm{~K}$ to
Talble 1

Correlation times $\tau_{\theta, \text { eff }}^{(2)}$ of liqiuid ammonia at $303 \mathrm{~K}$ and vapour press:ure calculated fro,m ${ }^{1: 4} \mathrm{~N}$ quadrupole relaxation times

\begin{tabular}{|c|c|c|c|}
\hline \multirow[b]{2}{*}{${ }^{14} \mathrm{NH}_{3}$} & \multirow{2}{*}{ 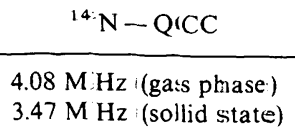 } & \multicolumn{2}{|c|}{$\left.\tau_{0, \text { eff }}^{(2)}{ }^{*}\right)$} \\
\hline & & $\begin{array}{l}121(\mathrm{fs}) \\
\left.16: 8(\mathrm{fs})^{\mathrm{a}}\right)\end{array}$ & $\begin{array}{l}115(\mathrm{fs}) \\
160(\mathrm{fs})\end{array}$ \\
\hline & & $\left.\downarrow^{* *}\right)$ & \\
\hline${ }^{14} \mathrm{ND}_{3}$ & $\begin{array}{l}4.08 \mathrm{M} \mathrm{Hz} \text { (gats phase) } \\
3.47 \mathrm{M} \mathrm{Hz} \text { (sollid state) }\end{array}$ & $\begin{array}{l}169(\mathrm{fs}) \\
23.3(\mathrm{fs})\end{array}$ & $\begin{array}{l}161(\mathrm{fs}) \\
\left.223(\mathrm{fs})^{\mathrm{b}}\right)\end{array}$ \\
\hline
\end{tabular}

a) Estimated from $T_{1}$-data cof Ref. [3]

b) Estimated from $T_{1}$-daata of RRef. [8]

*) $T=303 \mathrm{~K}$.

**) Transformed by appliicattion of Eq. (5) (see Atkins et al. [8]).

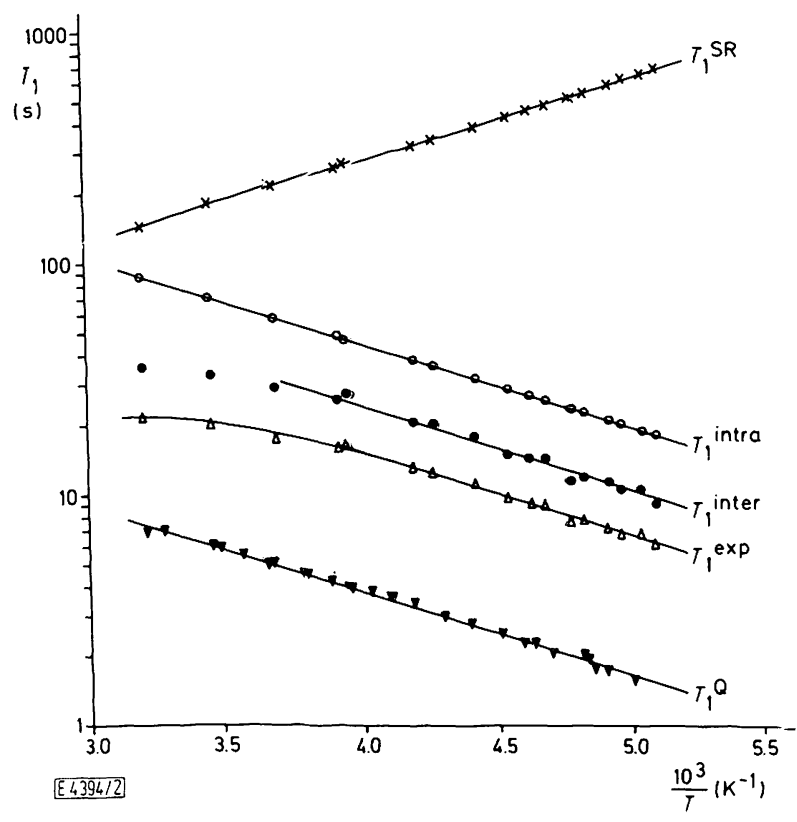

Fig. 2

Longitudinal relaxation times $\left(T_{1}\right)$ of the protons in $\mathrm{NH}_{3}\left(T_{1}^{\text {exp }}\right)$ and $\mathrm{ND}_{3}\left(T_{1}^{\mathrm{Q}}\right)$ at vapour pressure as function of the reciprocal temperature.

$T_{1}^{\text {intra }}$ intramolecular dipole-dipole relaxation rates of the protons in $\mathrm{NH}_{3}$.

- $T_{1}^{\text {inter }}$ intermolecular dipole-dipole relaxation rates of the protons in $\mathrm{NH}_{3}$.

$\times T_{1}^{\mathrm{SR}}$ spin rotation contribution to the experimental proton $T_{1}$. Details of the separation procedure for the different contributions to the experimental proton $T_{1}$ are given in the theoretical section (Eqs. (4), (5), and (6)]

$311 \mathrm{~K}$ for $\mathrm{NH}_{3}$ and $200 \mathrm{~K}$ to $310 \mathrm{~K}$ for $\mathrm{ND}_{3}$. The results are shown in Fig. 2 together with the different components contributing to the observed relaxation time. The separation has been effected as described before. It should be noticed that the intermolecular relaxation rate amounts to about $60 \%$ of the measured rate and is the most effective relaxation mechanism. This conclusion is in accord with the result obtained by Powles et al. $[6,7]$. However it depends strongly on the choice of the QCC. The correlation times extracted from these data are collected in Table 2. No definite decision can be made about the appropriate model of molecular motion in the case of liquid ammonia. Inspection of the correlation between $\tau_{\theta}^{*}$ and $\tau_{J}^{*}$ given by Powles and Rickayzen 


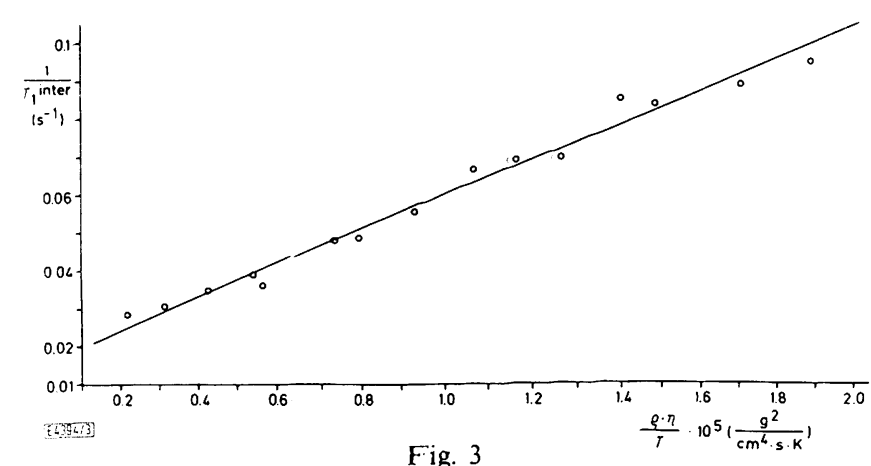

Plot of $\left(T_{1}^{\text {inter }}\right)^{-1}$ at vapour pressure ve;rs. $(\rho \cdot \eta) / T$ according to Eq. (2). $\rho=$ density., $\eta=$ dlynamic viscosity

T:able 2

Correlation times $\tau_{\theta e \text { if }}^{(2)}$ and $\tau_{3}$ of liquid $\mathrm{NH}_{3}$ at vapour pressure calculated by applicattion of Eq:s. (4), (5) and (6). The experimental intermolecular relaxation rates $\left(1 / T_{1}^{\text {inter }}\right)_{\text {exx }}$ give:n are the difference between $\left(1 / T_{1}^{\text {intra }}+1 / T_{1}^{\text {S:R }}\right)$ and the experimental $1 / T_{1}$. The calculated intermolecular relaxation ra.tes were de:termined with Eq. (2)

\begin{tabular}{lllll}
\hline$T(\mathrm{~K})$ & $\tau_{\theta, \text { eff }}^{(2)}(\mathrm{fis})$ & $\tau_{J}(\mathrm{fs})$ & $\begin{array}{c}\left(\frac{1}{T_{1}^{\text {initer }}}\right. \\
\left(\mathrm{s}^{-1}\right)\end{array}$ & $\left.\begin{array}{c}\left(\frac{1}{T_{\text {exp }}}\right. \\
T_{1}^{\text {inter }} \\
\left(\mathrm{s}^{-1}\right)\end{array}\right)$ \\
\hline 197 & 713 & 2.99 & 0.108 & \\
199 & 683 & 3.09 & 0.094 & \\
202 & 641 & 3.25 & 0.095 & 0.106 \\
204 & 615 & 3.35 & 0.089 & 0.096 \\
208 & 568 & 3.56 & 0.083 & 0.083 \\
210 & 547 & 3.66 & 0.085 & 0.078 \\
214 & 507 & 3.88 & 0.070 & 0.071 \\
217 & 480 & 4.04 & 01.069 & 0.066 \\
221 & 447 & 4.26 & 01.066 & 0.059 \\
227 & 404 & 4.59 & 0.055 & 0.053 \\
235 & 356 & 5.03 & 0.049 & 0.045 \\
239 & 335 & 5.25 & 0.048 & 0.041 \\
253 & 276 & 6.02 & 0.036 & 0.032 \\
255 & 269 & 6.13 & 0.039 & 0.030 \\
270 & 224 & 6.95 & 0.035 & 0.024 \\
288 & 184 & 7.93 & 0.031 & 0.018 \\
311 & 148 & 9.14 & 0.028 & 0.012 \\
\hline
\end{tabular}

[27] for different models shows that almost all $\tau_{\theta, \text { eff }}^{(2) *}$ fall into the region, where the Hubbard relation holds. This renders rotational diffusional motion to be an adequate description for the dynamic behaviour of the ammonia molecules over the temperature range studied.

Fig. 3 shows the dependence of $\left(1 / T_{1}\right)_{\text {inter }}$ on $(\rho \cdot \eta) / T$. As can be seen, the linear dependence which is predicted 'by Eq. (2) holds over the whole temperature range. Included in Table 2 are also the intermolecular relaxation rates calculated with Eq. (2). The agreement at low temperatures is reasonably good, the discrepancy at higher temperatures may be due to the separation procedure and to the simplicity of the underlying theoretical model. Concerning the anisotropy of the rotational motion of liquid ammonia we have estimated $\tau_{\text {, }}$ and $\tau_{\|}$for $\mathrm{ND}_{3}$ from the ${ }^{14} \mathrm{~N}-T_{1}$ data from Atkins et al. [8] and our deuteron $T_{1}$-data. At $T=303 \mathrm{~K}$ we get with QCC $\left({ }^{2} \mathrm{H}\right)=210 \mathrm{kHz}$ and $\mathrm{QCC}\left({ }^{14} \mathrm{~N}\right)=3.47 \mathrm{MHz}$ and the equation $[12,28]$.

$$
\begin{aligned}
\tau_{\theta, \mathrm{e}: \mathrm{f}}^{(2)} & =\tau_{\lrcorner}\left[1-\frac{3(\rho-1)}{5+\rho} \sin ^{2} \beta\left(1-\frac{3(\rho-1)}{2(2 \rho+1)} \sin ^{2} \beta\right)\right], \\
\nu & =\frac{\tau_{\lrcorner}}{\tau_{\|}}
\end{aligned}
$$

where $\beta$ is the ngle between the symmetry axis of the field gradient tensonnd the symmetry axis of the diffusion tensor an almost isotric rotation, i. e. $\rho \simeq 1$.

\section{Pressure Deperence of the Relaxation Times}

The dynami behaviour of the ammonia molecules as a function of thelensity has been studied up to a pressure of $250 \mathrm{MPa}$ for bth liquid $\mathrm{NH}_{3}$ and $\mathrm{ND}_{3}$. In the case of the former the terperature has been varied from $213 \mathrm{~K}$ to $467 \mathrm{~K}$, whereasor the latter the temperature interval ranged from $213 \mathrm{~K}$ to $\Sigma 1 \mathrm{~K}$. The isotherms of the spin-lattice relaxation times are sown in Figs. 4 and 5. The effective orientational correlationtimes $\tau_{\theta \text {,eff }}^{(2)}$ for $\mathrm{ND}_{3}$ have been calculated assuming $\left.\mathrm{QCC}^{2} \mathrm{H}\right)=210 \mathrm{kHz}$; they are given in Table 3 .

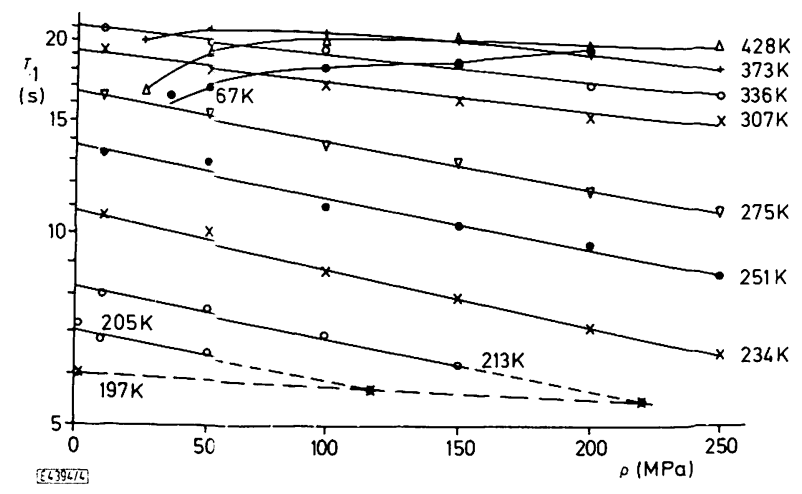

Fig. 4

Isotherms of the eperimental longitudinal proton relaxation times $\left(T_{1}\right)$ of liquid ammonia

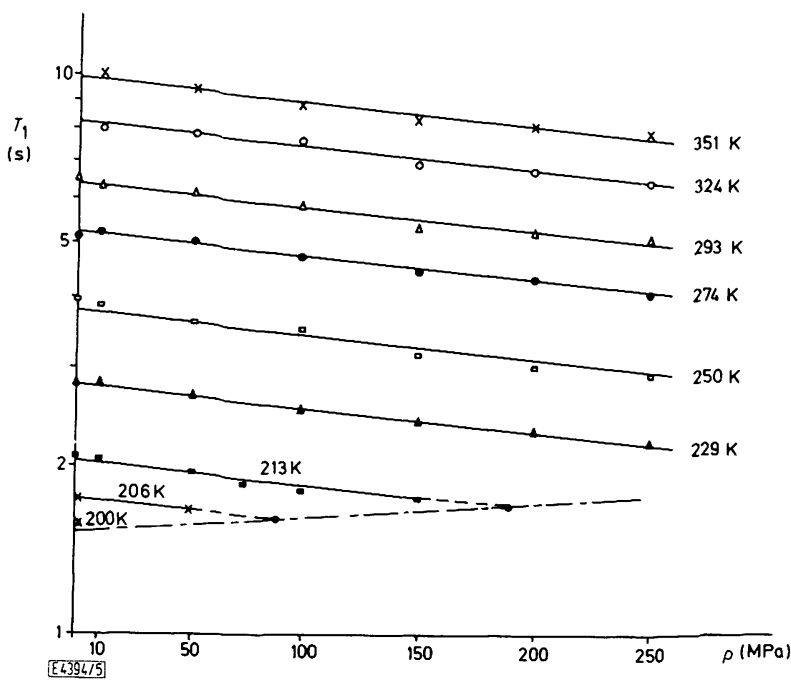

Fig. 5

Isotherms of the experimental longitudinal deuteron relaxation times $\left(T_{1}\right)$ of liquid deuteroammonia

In liquid ammonia at low temperatures $(T<350 \mathrm{~K})$ the separation procedure mentioned above was used to extract the different contributions to the total relaxation rate. The results are compiled for three pressures in Figs. 6-8. The effective orientational correlation times $\tau_{\theta, \text { eff }}^{(2)}$ for the intramolecular relaxation rate have been calculated from the $\tau_{\theta, \text { efr }}^{(2)}\left(\mathrm{ND}_{3}\right)$ with Eq. (5). However, the different geometrical 
Table 3

Pressure dependence of the correlation times $\tau_{\theta, \text { eff }}^{(2)}$ iliquid $\mathrm{ND}_{3}$ and $\tau_{J}$ in liquid $\mathrm{NH}_{3}$

\begin{tabular}{|c|c|c|c|c|c|c|}
\hline $\mathrm{ND}_{3}$ & \multirow[b]{2}{*}{$10 \mathrm{MPa}$} & \multicolumn{5}{|c|}{$\tau_{0 . \text { efr }}^{(2)}(\mathrm{fs})$} \\
\hline & & \multicolumn{5}{|c|}{$50 \mathrm{MPa} 100 \mathrm{MPa} 150 \mathrm{MPa}^{\prime} 00 \mathrm{MPa} 250 \mathrm{MPa}$} \\
\hline $213 \mathrm{~K}$ & 723 & 786 & 806 & 870 & 885 & 923 \\
\hline $229 \mathrm{~K}$ & 547 & 578 & 613 & 638 & 666 & 696 \\
\hline $250 \mathrm{~K}$ & 398 & 426 & 438 & 486 & 511 & 528 \\
\hline $274 \mathrm{~K}$ & 295 & 309 & 326 & 344 & 356 & 378 \\
\hline $293 \mathrm{~K}$ & 243 & 247 & 264 & 289 & 295 & 303 \\
\hline $324 \mathrm{~K}$ & 192 & 198 & 203 & 222 & 229 & 239 \\
\hline $351 \mathrm{~K}$ & 153 & 163 & 176 & 185 & 189 & 194 \\
\hline \multicolumn{2}{|l|}{$\mathrm{NH}_{3}$} & \multicolumn{5}{|c|}{$\tau_{J}(\mathrm{fs})$} \\
\hline & $10 \mathrm{MPa}$ & $50 \mathrm{MPa}$ & $100 \mathrm{MPa}$ & $150 \mathrm{MP}$ & $00 \mathrm{MP}$ & $250 \mathrm{MPa}$ \\
\hline $213 \mathrm{~K}$ & $3.80:$ & 3.49 & 3.40 & 3.15 & 3.10 & 2.97 \\
\hline $234 \mathrm{~K}$ & 4.92 & 4.68 & 4.44 & 4.11 & 4.03 & 3.86 \\
\hline $251 \mathrm{~K}$ & 5.86 & 5.58 & 5.28 & 4.90 & 4.79 & 4.60 \\
\hline $275 \mathrm{~K}$ & 7.18 & 6.83 & 6.45 & 6.00 & 5.88 & 5.64 \\
\hline $307 \mathrm{~K}$ & 8.84 & 8.40 & 7.96 & 7.40 & 7.25 & 6.95 \\
\hline $336 \mathrm{~K}$ & 10.3 & 9.78 & 9.20 & 8.57 & 8.34 & 8.07 \\
\hline
\end{tabular}

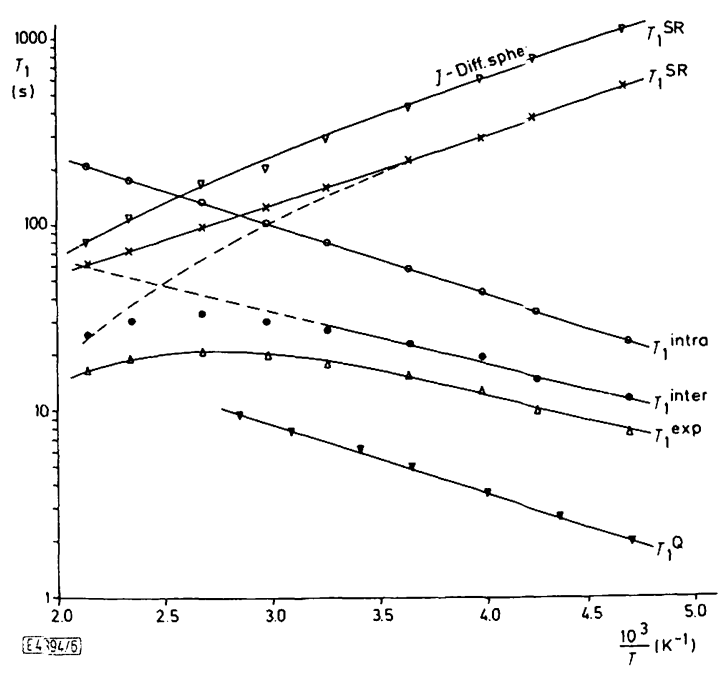

Fig. 6

Longitudinal relaxation times $\left(T_{1}\right)$ of the protons in $\mathrm{NH}_{3}\left(T_{1}^{\exp }\right)$ and $\mathrm{ND}_{3}\left(T_{1}^{\mathrm{Q}}\right)$ at $50 \mathrm{MPa}$ as function of the reciprocal temperature $O T_{1}^{\text {intra }}$ intramolecular dipole-dipole relaxaticn rates of the protons in $\mathrm{NH}_{3}$.

$T_{1}^{\text {inter }}$ intermolecular dipole-dipole relaxation rates of the protons in $\mathrm{NH}_{3}$.

$\times T_{1}^{\mathrm{SR}}$ spin rotation contribution to the experimental proton $T_{1}$.

$\nabla T_{1}^{\text {SR }}$ calculated from the spherical $J$-diffusion model (15).

Details of the separation procedure for the different contributions to the experimental proton $T_{1}$ are given in the theoretical section [Eqs. (4), (5), and (6)]

positions in the molecular frame of the corresponding vectors characterizing the interaction in question have been neglected. Since Eq. (5) has been experimentally verified in the case of nitrogen 14 in $\mathrm{NH}_{3}$ and $\mathrm{ND}_{3}$, where $\tau_{\theta \text {,eff }}^{(2)}=\tau^{(2,0)}$ it seems to be more appropriate to transform the spherical components instead of the effective correlation times. At pressures

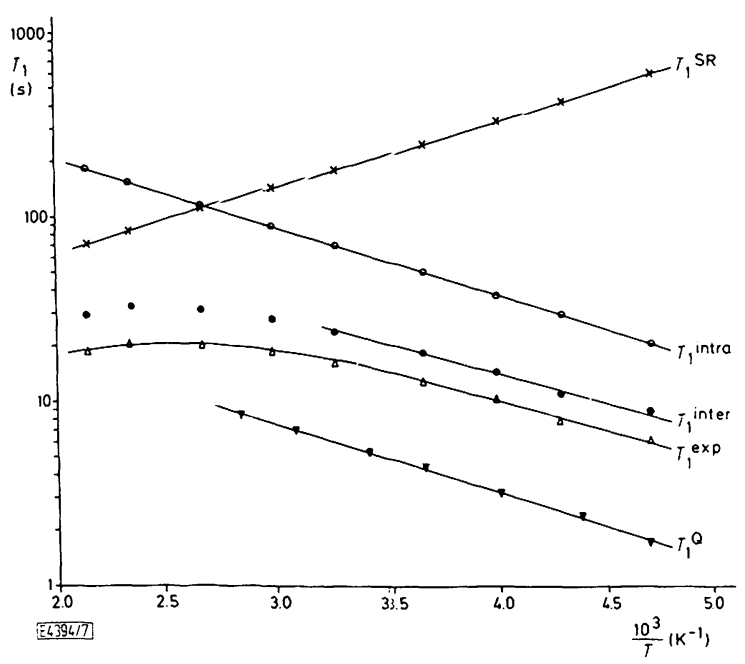

Fig. 7

Longitudinal relaxation times $\left(T_{1}\right)$ of the protons in $\mathrm{NH}_{3}\left(T_{1}^{\text {exp }}\right)$ and $\mathrm{ND}_{3}\left(T_{1}^{\mathrm{Q}}\right)$ at $150 \mathrm{MPa}$ as functiom of the reciprocal temperature. $O T_{1}^{\text {intra }}$ intramolecular dipole-dipiole relaxation rates of the protons in $\mathrm{NH}_{3}$.

- $T_{1}^{\text {inter }}$ intermolecular dipole-dipıle relaxation rates of the protons in $\mathrm{NH}_{3}$.

$\times T_{1}^{\mathrm{SR}}$ spin rotation contribution to the experimental proton $T_{\mathrm{i}}$. Details of the separation procedure for the different contributions to the experimental proton $T_{1}$ are given in the theoretical section (E.qs. (4), (5), and (6)]

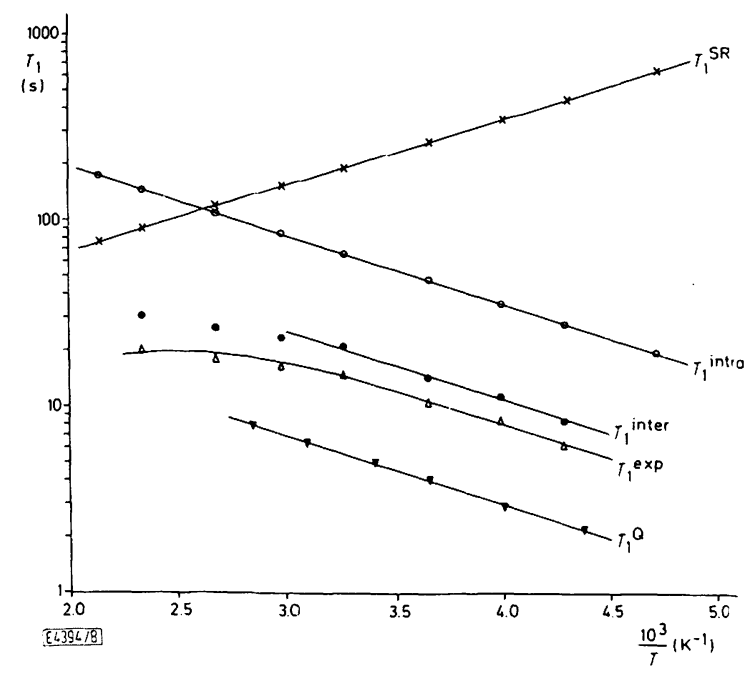

Fig. 8

Longitudinal relaxation times $\left(T_{1}\right)$ of the protons in $\mathrm{NH}_{3}\left(T_{1}^{\exp }\right.$, and $\mathrm{ND}_{3}\left(T_{1}^{\mathrm{Q}}\right)$ at $250 \mathrm{MPa}$ as function of the reciprocal temperature. $O T_{1}^{\text {intra }}$ intramolecular dipole-dipole relaxation rates of the protons in $\mathrm{NH}_{3}$.

$T_{1}^{\text {inter }}$ intermolecular dipole-dipole relaxation rates of the protons in $\mathrm{NH}_{3}$.

$\times T_{1}^{\mathrm{sR}}$ spin rotation contribution to the experimental proton $T_{1}$. Details of the separation procedure for the different contributions to the experimental proton $T_{1}$ are given in the theoretical section [Eqs. (4), (5), and (6)]

above saturation pressure only proton and deuteron $T_{1}$ measurements are available. It is therefore impossible to evaluate the spherical components $\tau_{\theta}^{(l, m)}$ of the effertive orientational correlation times. 
From the data presented lhere, it caannot be decided which motional model shiould be aupplied for the description of the dynamic behaviour of liquid! ammınia. However, only at the highest temperatures measured $(T>350 \mathrm{~K})$ the calculated effective correlation times $\tau_{\theta, \text { eff }}^{(2) *}$ leave the regime where all models merge into the small--step diffursion limit [27]. Because no independent determination off $\tau_{j j}^{* *}$ is possible with our measurements, no unambiguous deccision for one of the different models cian be made. One can only exclude the Ivanov-model $[27,29]$ since the low below the minimum value prossible in this model. In the low temperature region the small-step diffusion model should provide a reliable description and thius Eq. (6) was used to compute the anguliar momentum correlation times $\tau_{J}$; they are also given in Table 3.

Application of thigh pressure is sieen to influence both correlation times im an opposite way. While the orientational correlation time $\tau_{\theta ! \text {,eff }}^{(2)}$ increases with increasing pressure by $\sim 30 \%$, the angular momentum correllation time $\tau_{J}$ decreases over the same pressure range by the same amount. This behaviour only reflects the assiumed relationship $\tau_{\theta} \cdot \tau_{J}=$ const for an isotherm and is certainly in accord with small-step diffusive motion. The intermolecular relaxation rate is again the dominating relaxation me:chanism amounting from $\sim 67 \%$ at low pressures to $\sim 76 \%$ at the highest pressure. Thus the intermolecular dipole-dipole relaxation becomes more efficient with increasing presisure. This is to be expected since increasing density should increase the average number of next neighbours in a liquid and might probabily also decrease the distance $r_{12}$ between two protons on different mollecules thus enhancing the intermolecular dipole-dipole interaction. Increasing temperature causes the intermolecular relaxation rate to decrease. At the highest temperatures our separation procedure leads to an apparent increase for the efficiency of the intermolecular dipole-dipole interaction which is physically unacceptable and certainly an indication that the separation procedure applied fails at high temperatures and therefore small-step diffusion is no longer applicable. Assuming $T_{1}^{\text {inter }}$ to increase linearly on a semillog $1 / T$-plot one can conclude, that the spin-rotation contribution to the experimental relaxation rate becomes even more effective than is described by Eq. (3). It is probable that the $J$-diffusion model [15] predicts the true temperatu dependence as indicated by a computation of $T_{1}^{\mathrm{SR}}$ with arexpression given in Ref. [15], regarding for simplicity $\mathrm{NH}_{3} \mathrm{~S}$ a spherical molecule. However the absolute values of $T_{1}^{\text {SR }}$ re much to high to remove the apparent maximum in e $T_{1}^{\text {inter }}$ versus $T^{-1}$ isobares. In order to decide this quaion it would be necessary to measure the proton spin-latee relaxation times in $\mathrm{NH}_{3}$ at higher temperatures where thspin-rotation mechanism becomes even more dominating. $T_{2}$ high temperatures necessary cannot be obtained with ie variable temperature unit of our present spectrometer.

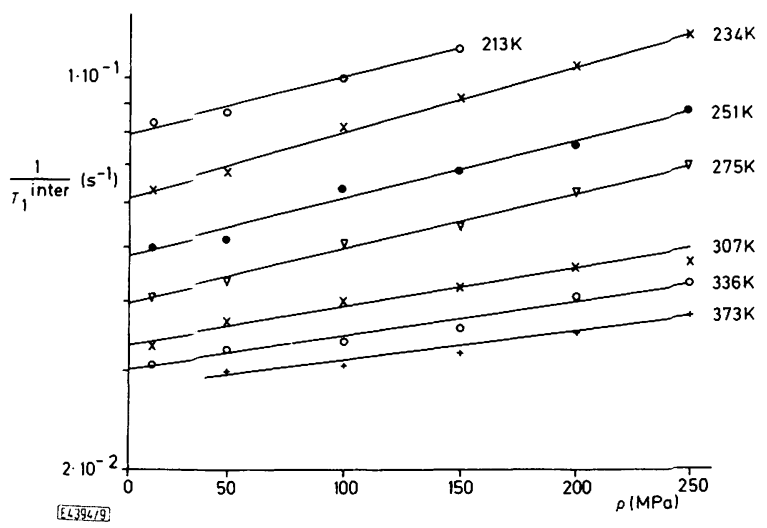

Fig. 9

Pressure dependece of the intermolecular dipole-dipole relaxation rates in $\mathrm{NH}_{3}$

The dynamic processes underlying the intra- and intermolecular relaxaion mechanisms are normally considered as thermally activaed. The corresponding activation cnergies and activation vdumes can be calculated from the respective semilog plots of $\tau_{\theta}$ versus temperature and pressure (Figs. 5 and 9). These parameters are collected in Table 4. All activation energis derived are significantly higher than $k T$, showing that the reorientation processes in liquid ammonia can be described is activated processes. It is generally assumed that the intramdecular dipole-dipole relaxation process is determined by the rotatoric reorientation of the molecules. The activation energy at constant pressure of $7 \mathrm{~kJ} \cdot \mathrm{mol}^{-1}$ for this process is to se compared with the corresponding results

Table 4

Activation energies and activation volumes calculated from the isobars, isochos or isotherms of the relaxation rates

Activation energies

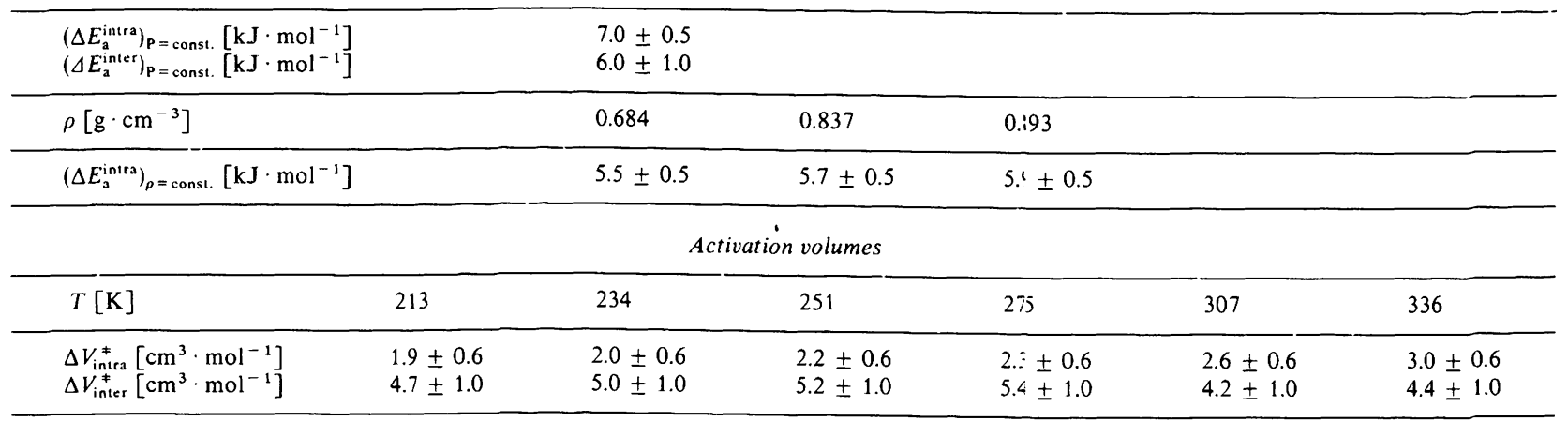


for water, where values around $14 \mathrm{~kJ} \cdot \mathrm{mul}^{-1}$ re derived [30] and for liquid hydrogensulfide [31] rhic yields $\sim 3 \mathrm{~kJ}$. $\mathrm{mol}^{-1}$. The comparison of these three mo:ules of similar size and moment of inertia shows that is liqd ammonia the hydrogen bonding between next neigbors hinders the reorientational process. This explanationlen further support from the activation energies at constait disity of $\sim 6 \mathrm{~kJ}$. $\mathrm{mol}^{-1}$. The ratio of the two activatior engies is $\sim 0.8$ in ammonia, while in normal liquids a rati arınd 0.5 is found [32]. The intermolecular relaxation rateis ninly determined by translational processes, the activation ergy derived for these processes is also very close to th: vae found for the rotation of a molecule. This indicates aga that identical activated processes which might tentatvel be explained as the breaking of a hydrogen bond are resonsible for this type of molecular motion.

From the isothermal pressure depenlens of the different relaxation processes the activation volıme $\Delta V^{\ddagger}$ defined by

$\Delta V^{\ddagger}=-R T\left(\frac{\partial \ln T_{1}}{\partial P}\right)_{T}$

can be derived. $\Delta V^{\neq}$is generally takenas a qualitative measure of the space required by a mlecle to reorient or translate to a new position in the cage ff it next neighbours. $\Delta V^{\neq}$for the rotational processes increises ontinuously with rising temperature. The same behavioir vuld be expected for the intermolecular term and the apprent decrease of $\Delta V_{\text {inter }}^{ \pm}$found for the $307 \mathrm{~K}, 336 \mathrm{~K}$, ind $373 \mathrm{~K}$ isotherms might, as mentioned in the previous ectin, be an artefact of our separation procedure. All activitio volumes derived for the intramolecular relaxation rate ae sinificantly smalle: than the corresponding values for the in:rmolecular term, thus indicating that the translational dfusion is slowed down faster with increasing density thn the rotational diffusion.

\section{Conclusions}

In the temperature range below $350 \mathrm{~K}$ the small-step diffusion model seems to provide an aleqate description of the dynamics of liquid ammonia. Due to ne limited temperature range of the present investigation $p$ decision can be made about the appropriate model for :he nolecular mobility at higher temperatures. Compared to vate at room temperature the reorientational correlation tims found in liquid ammonia are more than an order of nagnitude shorter. Furthermore the temperature dependence if $\tau_{\theta, \text { eff }}^{(2)}$ in ammonia is much smaller than in water. While in supercooled $\mathrm{D}_{2} \mathrm{O}$ $\tau_{\theta \text {, eff }}^{(2)}$ increases between $200 \mathrm{~K}$ and 30 ) $\mathrm{K}$ by three orders of magnitude $[30,35]$, the corresponding change in liquid $\mathrm{ND}_{3}$ is only a factor of 5. This indicats a much weaker intermolecular interaction than derved for the strongly hydrogen-bonded water. That some hydrigen bonding must exist in liquid ammonia becomes howerer evident from a comparison between the activation param:ters derived for the rotational and translational diffusion in $\mathrm{NH}_{3}$ and $\mathrm{H}_{2} \mathrm{~S}$. In the latter liquid the activation energie for the rotational and translational motion differ significantly [31] while in $\mathrm{NH}_{3}$ the isobaric and isochoric activation energies for the rotation as well as the corresponding energy for translational diffusion are very simila.r. Further evidence for this conclusion can be drawn from the low vallue of the deuteron QCC of $210 \mathrm{kHz}$ derived for liquicd $\mathrm{ND}_{3}$ as compared to the result observed in the gas of $282 \mathrm{kHIz}$ resp. the solid of $156 \mathrm{kHz}$. An increase of $80 \%$ for the QCC in $\mathrm{ND}_{3}$ in going from the solid state to the gas is comparablie to the increase of the QCC of $\mathrm{D}_{2} \mathrm{O}$ from ice to water vapoiur of $+66 \%$. In non hydrogenbonded liquids as for imstance $\mathrm{D}_{2} \mathrm{~S}[36,37]$ this change amounts only to $-4 \%$.

The radial distribution function of liquid $\mathrm{NH}_{3}$ derived from X-ray scattering [2] revreals approximately 12 next neighbours around a central molecule. This is in marked contrast to the result found in liquid water where the approximately tetrahedral symmetry of the ice crystal appears to be locally preserved and the central molecule is only surrounded by $\sim 4.4$ next nesighbours. Considering these observations, it is thus not surporising that the reorientational correlation times $\tau_{\theta, \text { eff }}^{(2)}$ in liquid ammonia increase continuously with pressure in the pressure range studied and reveal none of the anomalies cobserved at low temperatures and pressures in water.

The technical support by Mr. R. Knott and Mr. S. Heyn is gratefully acknowledged.

This work was supported by grants from the Deutsche Forschungsgemeinschaft and the Fonds der Chemischen Industrie.

\section{Refercences}

[1] C. N. R. Rao, in: F. Framks, ed., Water - A Comprehensive Treatise, Vol. 1. p. 93 ff., Plenum Press, New York 1972.

[2] A. H. Narten, J. Chem. Phys.. 66, 3117 (1977).

[3] J. L. Carolan and T. A. Scott, J. Magn. Reson. 2, 243 (1970).

[4] W. M. Litchman and M. A.lei, Jr., J. Chem. Phys. 56, 5818 (1972).

[5] W. G. Schneider, Symposium on Hydrogen Bonding, Ljubljana 1957.

[6] J. G. Powles and M. Rhodes., Mol. Phys. 12, 399 (1967).

[7] D. W. G. Smith and J. G. Powles, Mol. Phys. 10, 451 (1966).

[8] P. W. Atkins, A. Loewenstein, and Y. Margalit, Mol. Phys. 17, 329 (1969).

[9] J. G. Powles, M. Rhodes, and J. H. Strange, Mol. Phys. 1/. 515 (1966)

[10] G. Völkel, E. Lang. and H..-D. Lüdemann, Ber. Bunsenges. Phys. Chem. 83, 722 (1979).

[11] A. Abragam, The Principles of Nuclear Magnetism, Orford University Press 1961.

[12] H. W. Spiess, in: P. Diehl, E. Fluck, R. Kosfeld, eds., N.MRBasic Principles and Progress, Vol. 15, p. 55 ff., Springer Verlag. Berlin 1978.

[13] W. A. Steele, in: I. Prigogine and S. A. Rice, eds., Advances in Chemical Physics, Vol. 34, p. 1 ff., Wiley, New York 1976

[14] M. D. Zeidler, Ber. Bunsenges. Phys. Chem. 75, 229 (1э71).

[15] R. E. D. McClung, Advances in Molecular Relaxation and Interaction Processes 10,83 (1977).

[16] P. S. Hubbard, Phys. Rev. 131, 275 (1963)

[17] H. C. Torrey, Phys. Rev. 92, 962 (1953).

[18] J. F. Harmon and B. H. Muller, Phys. Rev. 182, 400 (1†69).

[19] B. H. Muller, Phys. Lett. 22. 123 (1966).

[20] J. F. Harmon, J. Magn. Reson. 31, 411 (1978).

[21] R. L. Cook and F. C. DeLucia, Am. J. Phys. 39, 1433 (1971).

[22] C. H. Wang, J. Magn. Reson. 9, 75 (1973).

[23] R. M. Garvey, F. C. DeLucia, and J. W. Cederberg, Mol. Phys. 31,265 (1976). 
[24] Landolt-Börnstein, New serie:s, K.-H. Hellwege, A. M. Hellwege, eds., Springer Verlag, Berlin 1974, Vol. II/6, p. 413.

[2.5] P. S. Hubbard, Phys. Rev. 1.31, 1155 (1963).

[26] D. W. Sawyer and J. G. Powles. Mol. Phys. 21, 83 (1971).

[27] J. G. Powles and G. Rickkayzen, Mol.. Phys. 33, 1207 (1977)

[2:8] W. T. Huntress, Jr., in: J. 'S. WNaugh. ed.., Advances in Magnetic Resonance, Vol. 4, p. 1 ff.., Alcademic Press, New York 1970.

[29] E. N. Ivanov, Soviet Physs. JETP 1.8, 1041 (1964).

[3(O] E. Lang and H.-D. Lüdermanin, J. Chern. Phys. 67, 718 (1977).
[31] J. Hauer, riplcmarbeit, Universität Regensburg 1978.

[32] J. Jonas, T De'ries, and D. J. Wilbur, J. Chem. Phys. 65, 583 (1976).

[33] E. Lang ant H.D. Lüdemann, to be published.

[34] F. C. DeLcia ind J. W. Cederberg, J. Mol. Spectrosc. 40, 52 (1971).

[35] D. E. O'Rely and J. H. Eraker, J. Chem. Phys. 52, 2407 (1970).

(Eingegangen am 27. Juli 1979, E 4394 endgültige Fassung am 18. August 1979) 